\title{
1 Biotic interactions and climate in species distribution modelling
}

3 Daniel P. Bebber*, Department of Biosciences, University of Exeter, Stocker

4 Road, Exeter EX4 4QD, UK

5 Sarah J. Gurr, Department of Biosciences, University of Exeter, Stocker Road,

$6 \quad$ Exeter EX4 4QD, UK

7

8 Summary

$9 \quad$ Species have preferred environmental niches ${ }^{1}$ and their geographical

10 distributions respond to global climate change ${ }^{2}$. Predicting range shifts under

11 climate change has profound implications for conservation of biodiversity ${ }^{3}$,

12 provision of ecosystem services, and in the management of invasive species ${ }^{4}$.

13 Species distribution modelling (SDM) has largely focussed on climate variations,

14 but biotic interactions, such as predation and competition, can alter potential

15 distributions ${ }^{5,6}$ and affect migration rates ${ }^{7}$. However, a lack of data on biotic

16 interactions has restricted consideration of these factors for many species ${ }^{1}$.

17 Here, we compare the power of biotic and climatic factors as predictors of global

18 distributions of hundreds of crop pests and pathogens (CPPs), for which host

19 preferences are known. We show that host availability is a more important

20 predictor of endobiotic pathogen distributions (fungi, oomycetes, bacteria, viruses

\footnotetext{
* Corresponding author: D.Bebber@exeter.ac.uk
} 
21 and nematodes) than of epibiotic pest distributions (insect herbivores).

22 Conversely, climatic variables are better predictors of epibiotic pest

23 distributions. These results are robust to statistical controls for varying

24 observational capacity among countries. Our findings demonstrate that life

25 history affects global scale species distributions and that SDM should

26 incorporate biotic interactions as well as climate.

29 The strong influence of climate on species distributions has motivated the field of 30 Species Distribution Modelling (SDM) ${ }^{8}$. In SDM, species' climatic preferences 31 and tolerances, derived by experiment or inferred from observed distributions, 32 are used to project how the location of suitable habitat may change in the future 33 8. While the methods and approaches for climate-driven SDM have invoked some 34 controversy ${ }^{9,10}$, there has been little discussion of the other factors that 35 determine where species may be found ${ }^{1,6}$. Among these are various biotic interactions, such as the availability of food or interference from competitors ${ }^{1,5}$,

37 and the ability of species to reach a suitable habitat - illustrated by the rapid 38 global redistribution of species by human activities in recent times ${ }^{11}$.

39 The importance of biotic interactions and dispersal in determining species 40 distributions has been distilled in the Biotic-Abiotic-Migration (BAM)

41 framework ${ }^{1,6}$. Species dispersal limitation has received considerable attention, 42 for example, certain species seem unable to keep up with the rate of climatic 
43 change ${ }^{12}$. In contrast, the role of biological factors in modifying distributions is

44 poorly understood, because the complex network of interactions that could

45 influence species distribution is largely unknown ${ }^{1}$. While examples of the roles

46 that biotic factors can play in moderating migration rates, and thus non-

47 equilibrium distributions, are beginning to accumulate ${ }^{7}$, there remains a lack of

48 synthesis of the general patterns and principals governing the importance of

49 biological interactions in SDM. Soberon and Nakamura ${ }^{1}$ state that "Without a

50 much larger empirical database about such [biotic] factors, the relative roles of

51 [abiotic] and [biotic] factors in determining distributions would be difficult to

52 assess".

53 Here, we address this question using the distributions of the pests and

54 pathogens that attack agricultural crops. Crop pests and pathogens (CPPs)

55 comprise a diverse group of organisms, including thousands of species and

56 pathotypes of fungi, oomycetes, bacteria, viruses, nematodes, insects and other

57 arthropods ${ }^{11,13}$. CPPs inhabit simplified agricultural ecosystems in which

58 intensive management practices have reduced biological diversity and physical

59 complexity. Therefore, the network of biotic interactions in which CPPs take part

60 is also likely to be simplified and tractable. CPPs are of great socioeconomic

61 importance, and hence attract research interest and efforts to catalogue their

62 biology and monitor their distributions ${ }^{11}$. The distributions of many of their host

63 plants are also known ${ }^{14}$. CPPs may therefore allow the relative importance of

64 abiotic and biotic variables in determining distributions to be quantified. 
65 Simply finding that host availability and climatic variables are predictors of CPP

66 presence would be uninformative because CPPs are likely to have similar

67 climatic niches to their hosts, and therefore the two predictors will be correlated.

68 Rather, we compared the relative predictive power of abiotic and biotic variables

69 for CPPs that are likely to differ in the strength of their biological interaction

70 with, and dependence upon, their hosts. Invasive endobiotic pathogens such as

71 the viruses, bacteria, oomycetes and fungi are "buffered" to some degree from the

72 external environment by their respective hosts. Pathogen growth rates, for

73 example, may be better described by temperatures within host plant tissues

74 rather than by external air temperature ${ }^{15}$. Thus, their presence should be more

75 dependent upon host distributions than are epibiotic arthropod pests, which are

76 likely to be more directly exposed to the weather.

77 Generalized Additive Models (GAMs) fitted to presence/absence data explained a mean of $16.8 \pm 0.3 \%$ of the deviance using host availability, $27.8 \pm 0.4 \%$ using

79 climatic variables, and $37.7 \pm 0.5 \%$ using both host and climate predictors.

80 There was significant variation among CPP taxonomic groups, with biotic

81 variables being better predictors for pathogens than for pests (Fig. 1). The

82 difference $D_{\text {diff }}$ in the fraction of deviance explained between climate $\left(\mathrm{D}_{\text {clim }}\right)$ and

83 host (Dhost) was $6.9 \pm 1.5 \%$ for pathogens, and $15.6 \pm 0.9 \%$ for pests (mixed

84 effects model with random intercepts per taxonomic group, $\mathrm{F}_{1,8}=17.54, \mathrm{p}=$

85 0.003). There was no significant influence of host number on the difference in

$86 \mathrm{D}_{\text {diff }}$ between pests and pathogens (mixed effects model including log host

87 number, $\left.\mathrm{F}_{1,1029}=-0.49, \mathrm{p}=0.63\right)$. 

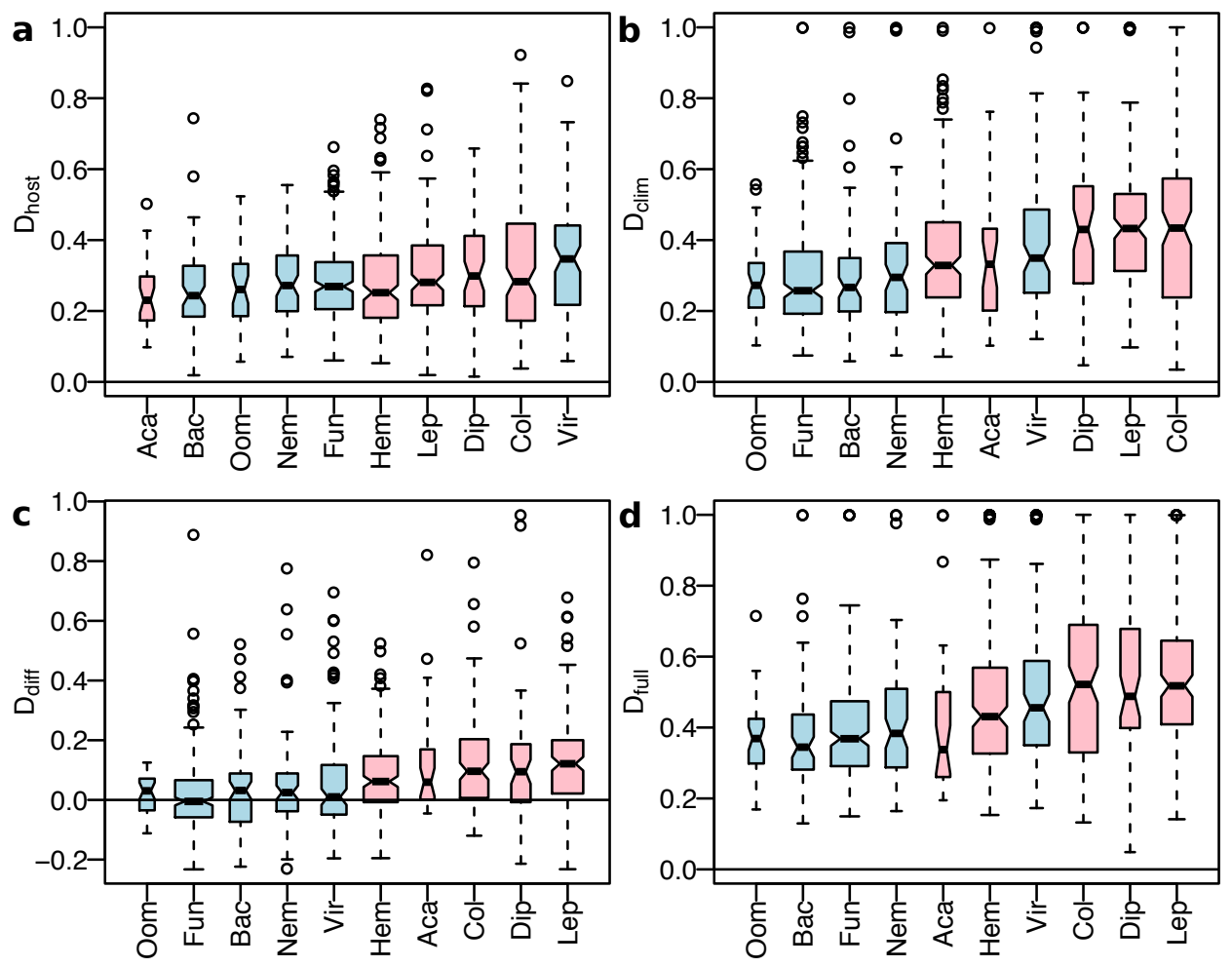

Fig 1. Explanatory power of GAMs. a. Deviance explained by host availability,

Dhost, for each CPP category in order of mean. Pathogens are blue and pests pink.

b. Deviance explained by the climate model, $D_{\text {clim. }}$ c. Difference between $D_{\text {clim }}$ and in the boxes do not overlap between categories, this indicates a significant sample size.

$D_{\text {full }}$ declined with the number of host plants per CPP (Fig. 2; mixed effects model with random intercepts per taxonomic group, $\mathrm{F}_{1,1028}=34.5, \mathrm{p}<0.001$ ) and was greater for pests compared with pathogens $\left(\mathrm{F}_{1,8}=8.0, \mathrm{p}=0.022\right)$, but there was no difference between pests and pathogens in the relationship to host number 
$101 \quad\left(\mathrm{~F}_{1,1028}=0.022, \mathrm{p}=0.88\right)$. The full model explained nearly all the deviance $\left(\mathrm{D}_{\text {full }}>\right.$

102 0.95) for 75 CPPs with restricted distributions (mean of 21.1 presences). Similar

103 results were obtained using models for which observational bias among countries

104 was modelled as a modification of absence data related to national scientific

105 output ${ }^{13}$ (Supplementary Fig. 1).

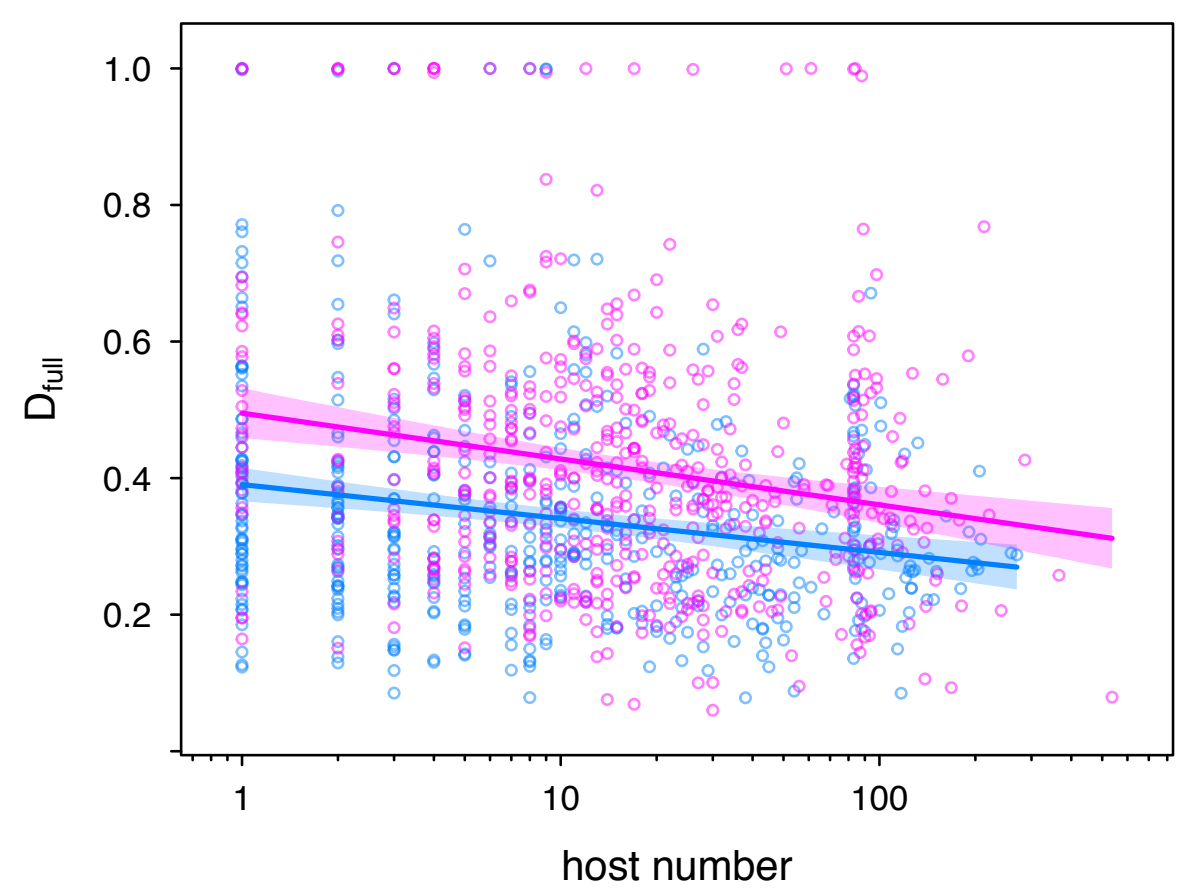

107 Fig. 2. Model explanatory power declines with host range. Open circles show

108 values for individual pests (pink) and pathogens (blue). Bold lines show linear model fits, with $95 \%$ Confidence Limits shaded.

111 We found that host availability is a better predictor of species distributions for

112 endobiotic pathogens than it is for epibiotic pests. Thus, variation in the strength

113 of interaction between consumers and their host plants translates to 
114 biogeographic patterns at the global scale. Araújo and Luoto ${ }^{5}$ first tested the

115 hypothesis that biotic interactions can limit species distributions at

116 macroecological scales, showing that the distribution of the clouded Apollo

117 butterfly (Parnassius mnemosyne) in Europe is controlled by the distribution of

118 host plants (Corydalis spp.), as well as by climate. The butterfly is threatened

119 across much of its geographic range and has been the subject of much

120 conservation research, hence its biotic niche has been largely described. For

121 many other wild species such information is unavailable, and so we turned to

122 CPPs whose biotic interactions are better described. While SDM of CPPs in

123 relation to climate is relatively common ${ }^{16,17}$, combined modelling of both pest

124 and hosts has been rarely attempted. For example, projections of future habitat

125 suitability for the soybean (Glycine max) and the bean leaf beetle (Cerotoma

126 trifurcata) indicate that host distribution will limit the potential pest

127 distribution ${ }^{18}$. The soybean shows wide climatic tolerance, but the temperature

128 range of the beetle, a generalist feeding on numerous hosts, is even wider.

129 Detecting any influence of host availability is perhaps surprising because we

130 expect CPPs to be highly-adapted to their hosts and likely to evolve to match the

131 host's climatic niche. Therefore, the biotic and climatic predictors should be

132 highly correlated. For example, populations of the temperate climate wheat

133 pathogen Zymoseptoria tritici isolated from warmer regions show genetically-

134 determined variation in optimal growth temperatures and temperature

135 responses ${ }^{19}$, and are therefore able to match the local climate experienced by the

136 host. However, crops are not grown in all areas that are climatically suitable,

137 allowing statistical models to partition the influence of these predictors. In 
138 addition, the example of the bean leaf beetle illustrates that host and pest

139 climatic niches may not correspond exactly 18.

140 Host crops present a local environment that is substantially different from the

141 external climate, represented by climatic variables used in SDM. Air

142 temperature and moisture vary through a crop canopy, and leaf temperatures

143 differ from air temperatures above the canopy depending upon insolation and

144 leaf water status ${ }^{20}$. Thus, CPPs will experience different microclimates from

145 those suggested by meteorological data, and endobiotic pathogens, in particular,

146 will be influenced by in planta conditions ${ }^{15}$. SDMs utilize meteorological data

147 and biologically relevant derivatives to estimate the climatic niche ${ }^{21,22}$, but for

148 endobiotic organisms it appears that the environment provided by the host crop

149 is as good a predictor of presence, albeit at a global scale, as is climate. Some

150 physiological models of pathogen risk explicitly consider the host environment.

151 For example, a model of overwinter survival by Phytophthora cinnamomi, under

152 oak bark, contains a transfer equation to calculate inner bark temperature from

153 air temperature ${ }^{23}$.

154 There was no significant relationship between host specificity (the number of

155 plant genera known to be attacked by a pest or pathogen) and the relative

156 explanatory power of biotic versus climatic predictors of species distributions. We

157 might expect that generalist CPPs would not be limited by host availability and

158 therefore respond more strongly to climate than specialist CPPs. However, the

159 area of agricultural land varies widely among countries, and even a generalist

160 able to consume any plant would therefore still be more likely found in a country 
161 with a large cropping area. Therefore, climatic predictors need not be more

162 important for generalist CPPs. We did find that the distributions of generalist

163 CPPs are less predictable overall, which suggests that their establishment is less

164 hindered by the physical environment and may be determined by other factors,

165 such as anthropogenic dispersal.

166 Our analysis supports the argument that biotic interactions should be included

167 in SDM 1,5,18, particularly where strong dependence on other species is known or

168 suspected. Plant viruses are obligate parasites and arguably the most strongly

169 host-dependent of all CPPs - viral distributions appeared to be most strongly

170 determined by host availability. However, the expected role of climate versus

171 host availability in determining viral distributions is complicated by the fact that

172 many plant viruses are dependent upon insect vectors, particularly aphids, for

173 transmission ${ }^{24}$. Aphids transmit nearly 300 plant viruses, and their populations

174 respond strongly to weather ${ }^{25}$. Therefore the distribution of viruses could partly

175 reflect the climatic controls on their vectors. However, Hemiptera are the most

176 widespread of all insect pest groups ${ }^{11}$, with the cotton aphid Aphis gossypii, the

177 green peach aphid Myzus persicae, and the silverleaf whitefly Bemisia tabaci

178 most cosmopolitan. Each of these species transmits many viruses. Host crops

179 may thus be the limiting factor in viral distributions where the vector is

180 widespread.

181 We investigated only one biotic factor, host availability, which we propose is

182 most important for CPPs. Predators of CPPs, for example, appear to be most

183 important within the native range, their prey finding 'enemy-free space' upon 
184 dispersal ${ }^{27}$. For wild species, many other interactions will determine the rate

185 and pattern of range shift in response to climate change ${ }^{7}$, and as empirical data

186 accumulate, knowledge of general principles governing range shifts will improve.

187 The importance of weather in determining the risk of CPP outbreaks has long

188 been recognized, resulting in numerous statistical and mechanistic models across

189 a wide range of spatiotemporal scales ${ }^{17}$. Researchers have recently begun to

190 investigate the distributional determinants of individual CPPs and how these

191 might develop as the climate changes. In many cases, the response of the pest is

192 modelled, but not the host crop ${ }^{28}$. This simplification could lead to serious errors

193 in projections if, for example, the pest and host crop occupy different climatic

194 niches ${ }^{18}$. Analyses incorporating both the pest and the host are rare, and even

195 these do not attempt to estimate the influence of dispersal limitation. We have

196 shown that fundamental differences in life history influence the relative

197 importance of biotic and abiotic factors in determining global distributions for a

198 class of organisms, CPPs, for which biotic niche information is available.

199 Accumulation of such data for wild species will help to improve SDM for

200 projections of how the biosphere is likely to change with global warming.

\section{Methods}

202 We compared the relative predictive power of abiotic (climate) and biotic (host

203 availability) variables on current species distributions by statistically modelling

204 the current global distributions of CPPs at national scale. We employed the

205 approach of 'surrogate hypotheses' 5 , whereby correlations in observational data

206 are tested in lieu of controlled experiments, which are difficult to conduct for 
207 macroecological processes. We compared the goodness-of-fit of biotic (host

208 availability) and abiotic (climate) variables to CPP presence-absence data at

209 country level (state level for the USA, Brazil, India, China and Australia) using

210 Generalized Additive Models (GAMs) with binomial errors ${ }^{29}$. The 'biotic model'

211 used the log-transformed areas of host crops for the CPP as predictors ${ }^{13}$; the

212 'climate model' used the climate in areas where the host crops are grown. The

213 'full model' used both biotic and climate variables. We obtained CPP presence

214 data, and known host plants, for each CPP from CABI with permission.

215 Taxonomic categories of CPPs for which fewer than twenty species were

216 available (Psocoptera, Thysanoptera) were omitted, as were CPPs present in ten

217 or fewer locations, leaving 1040 CPPs. We summed spatial distributions of major

218 crops from the MIRCA2000 database ${ }^{14}$ to country and state level, to match CPP

219 distribution data. For each country/state, we calculated the total production area

220 of each crop, and for each CPP we calculated the total production area for each

221 host crop. Host crop areas were log-transformed for fitting. We selected four

222 bioclimatic variables from the BIOCLIM database ${ }^{30}$ : mean annual temperature;

223 standard deviation of monthly temperature; mean annual rainfall; standard

224 deviation of monthly rainfall. We omitted the other BIOCLIM variables (annual

225 temperature range, precipitation of driest month, etc.) to avoid multi-

226 collinearity, as these derived variables are strongly correlated with those we

227 selected. Area-weighted means of these climatic variables were calculated for

228 crop-growing areas per region. GAMs were fitted using splines for each predictor

229 variable ${ }^{31}$. We analysed the difference between the fraction of deviance 
explained by the abiotic and biotic models to determine whether the relative

231 importance of host availability varied between pests and pathogens.

232 Available global CPP distribution data are biased by the varying abilities of

233 countries to detect, identify and report CPP presence. Richer countries at higher

234 latitudes tend to have better plant health reporting systems, and there is a

235 strong correlation between countries' scientific output and reported pest

236 incidence ${ }^{4,13}$. While presences reported in the CABI databases are likely to be

237 reliable due to stringent quality checking procedures, absences could be pseudo-

238 absences where a CPP is present but unreported. Let $d_{n}$ be the binary presence-

239 absence data for a pest or pathogen in each of $n$ geographical regions, and let $w_{n}$

240 be the corresponding confidence weighting for that region. Then the confidence-

241 weighted presence probabilities are

$242 \quad d_{n}^{w}=d_{n}+\frac{\left(1-2 d_{n}\right)\left(1-w_{n}\right)}{2}$

243 Our confidence in presences is absolute, i.e.

244 when $d_{n}=1, w_{n}=1$

245 Our confidence in absences varies as a fitted quadratic function of the number of

246 scientific publications per country between 1996 and $2012^{13}, s_{n}$, such that

247 when $d_{n}=0, w_{n}=-0.001085 \ln \left(s_{n}+1\right)+0.005074 \ln \left(s_{n}+1\right)^{2}$

248 Therefore, our confidence in absences from the USA is complete (the USA has

249 the largest scientific output), $w_{n}=1$, and $d_{n}^{w}=0$ when $d_{n}=0$. Our confidence in 
250 absences from the world's least developed countries is near zero, and $d_{n}^{w} \sim 0.5$.

251 This method is not equivalent to weighting observations by confidence, nor

252 including confidence as a predictor in the model, because the weighting only

253 affects our treatment of absences. We fitted fourth-order polynomials of the

254 predictor variables to $d_{n}^{w}$, minimizing $\chi^{2}$ of the difference between observed and

255 fitted values by optimization. Quadratic functions are often used in SDM 29, but

256 quartic functions allow a greater range of response profiles to be described,

257 without over-fitting. Presence-absence data are often fitted using Generalized

258 Linear Models or Generalized Additive Models ${ }^{5}$, but these methods require

259 errors distributions in the exponential family for maximum likelihood estimation

260 31. The data do not follow the binomial distribution, and we are therefore unable

261 to calculate likelihoods for fitted models, nor the various model selection metrics

262 based upon likelihood (e.g. AIC), using GLM or GAM. Instead, we obtained $\chi^{2}$

263 and AUC statistics for the biotic model, the climate model, and the combined

264 model. These values were then compared using linear models to investigate the

265 relative explanatory power of biotic and abiotic predictors.

\section{References}

267 1. Soberón, J. \& Nakamura, M. Niches and distributional areas: concepts, 268 methods, and assumptions. Proc. Natl. Acad. Sci. 106, 19644-19650 (2009).

269 2. Chen, I.-C., Hill, J. K., Ohlemüller, R., Roy, D. B. \& Thomas, C. D. Rapid 270 range shifts of species associated with high levels of climate warming. Science $271 \quad 333,1024-1026(2011)$. 
3. Hannah, L. et al. Protected area needs in a changing climate. Front. Ecol. Environ. 5, 131-138 (2007).

4. Bebber, D. P., Ramotowski, M. A. T. \& Gurr, S. J. Crop pests and pathogens move polewards in a warming world. Nat. Clim. Change 3, 985-988 (2013).

5. Araújo, M. B. \& Luoto, M. The importance of biotic interactions for modelling species distributions under climate change. Glob. Ecol. Biogeogr. 16, 743-753 (2007).

6. Soberón, J. Grinnellian and Eltonian niches and geographic distributions of species. Ecol. Lett. 10, 1115-1123 (2007).

7. Svenning, J.-C. et al. The influence of interspecific interactions on species range expansion rates. Ecography 37, 1198-1209 (2014).

8. Dormann, C. F. et al. Correlation and process in species distribution models: bridging a dichotomy. J. Biogeogr. 39, 2119-2131 (2012).

9. Kriticos, D. J., Le Maitre, D. C. \& Webber, B. L. Essential elements of discourse for advancing the modelling of species' current and potential distributions. J. Biogeogr. 40, 608-611 (2013).

10. Schymanski, S. J. et al. Process, correlation and parameter fitting in species distribution models: a response to Kriticos et al. J. Biogeogr. 40, 612-613 (2013).

291 11. Bebber, D. P., Holmes, T. \& Gurr, S. J. The global spread of crop pests and pathogens. Glob. Ecol. Biogeogr. 23, 1398-1407 (2014).

12. Devictor, V. et al. Differences in the climatic debts of birds and butterflies at a continental scale. Nat. Clim Change 2, 121-124 (2012). 
13. Bebber, D. P., Holmes, T., Smith, D. \& Gurr, S. J. Economic and physical determinants of the global distributions of crop pests and pathogens. New Phytol. 202, 901-910 (2014).

14. Portmann, F. T., Siebert, S. \& Döll, P. MIRCA2000—Global monthly irrigated and rainfed crop areas around the year 2000: A new high-resolution data set for agricultural and hydrological modeling. Glob. Biogeochem. Cycles 24, 1-24 (2010).

15. Bernard, F., Sache, I., Suffert, F. \& Chelle, M. The development of a foliar 303 fungal pathogen does react to leaf temperature! New Phytol. 198, 232-240

304 (2013).

16. Sparks, A. H., Forbes, G. A., Hijmans, R. J. \& Garrett, K. A. Climate change 306 may have limited effect on global risk of potato late blight. Glob. Change Biol. 20, 3621-3631 (2014).

17. Sutherst, R. W. Pest species distribution modelling: origins and lessons from history. Biol. Invasions 16, 239-256 (2014).

18. Berzitis, E. A., Minigan, J. N., Hallett, R. H. \& Newman, J. A. Climate and host plant availability impact the future distribution of the bean leaf beetle (Cerotoma trifurcata). Glob. Change Biol. n/a-n/a (2014). doi:10.1111/gcb.12557

19. Zhan, J. \& McDonald, B. A. Thermal adaptation in the fungal pathogen Mycosphaerella graminicola. Mol. Ecol. 20, 1689-1701 (2011). differentially-irrigated corn. Agric. Meteorol. 25, 207-217 (1981). 
21. Booth, T. H., Nix, H. A., Busby, J. R. \& Hutchinson, M. F. bioclim: the first species distribution modelling package, its early applications and relevance to most current MaxEnt studies. Divers. Distrib. 20, 1-9 (2014).

22. Kriticos, D. J. et al. CliMond: global high-resolution historical and future scenario climate surfaces for bioclimatic modelling. Methods Ecol. Evol. 3, $53-64(2012)$.

23. Marçais, B., Dupuis, F. \& Desprez-Loustau, M. L. Modelling the influence of winter frosts on the development of the stem canker of red oak, caused by Phytophthora cinnamomi. Ann. Sci. For. 53, 369-382 (1996).

24. Jones, R. A. C. Plant virus emergence and evolution: Origins, new encounter scenarios, factors driving emergence, effects of changing world conditions, and prospects for control. Virus Res. 141, 113-130 (2009).

25. Harrington, R. et al. Environmental change and the phenology of European aphids. Glob. Change Biol. 13, 1550-1564 (2007).

26. Bebber, D. P., Holmes, T. \& Gurr, S. J. The global spread of crop pests and pathogens. Glob. Ecol. Biogeogr. n/a-n/a (2014). doi:10.1111/geb.12214

27. Thomson, L. J., Macfadyen, S. \& Hoffmann, A. A. Predicting the effects of climate change on natural enemies of agricultural pests. Biol. Control 52, 296-306 (2010).

28. Bregaglio, S., Donatelli, M. \& Confalonieri, R. Fungal infections of rice, wheat, and grape in Europe in 2030-2050. Agron. Sustain. Dev. 33, 767-776 (2013).

29. Elith, J., Kearney, M. \& Phillips, S. The art of modelling range-shifting species. Methods Ecol. Evol. 1, 330-342 (2010). 
342 30. Hijmans, R. J., Cameron, S. E., Parra, J. L., Jones, P. G. \& Jarvis, A. Very

343 high resolution interpolated climate surfaces for global land areas. Int. J.

$344 \quad$ Climatol. 25, 1965-1978 (2005).

345 31. Wood, S. Generalized Additive Models: An Introduction with R. (CRC Press,

$3462006)$.

\section{Acknowledgements}

348 Data on pest and pathogen geographical distributions and host plants were

349 obtained with permission from CABI.

350

351 
353 Supplementary Figures
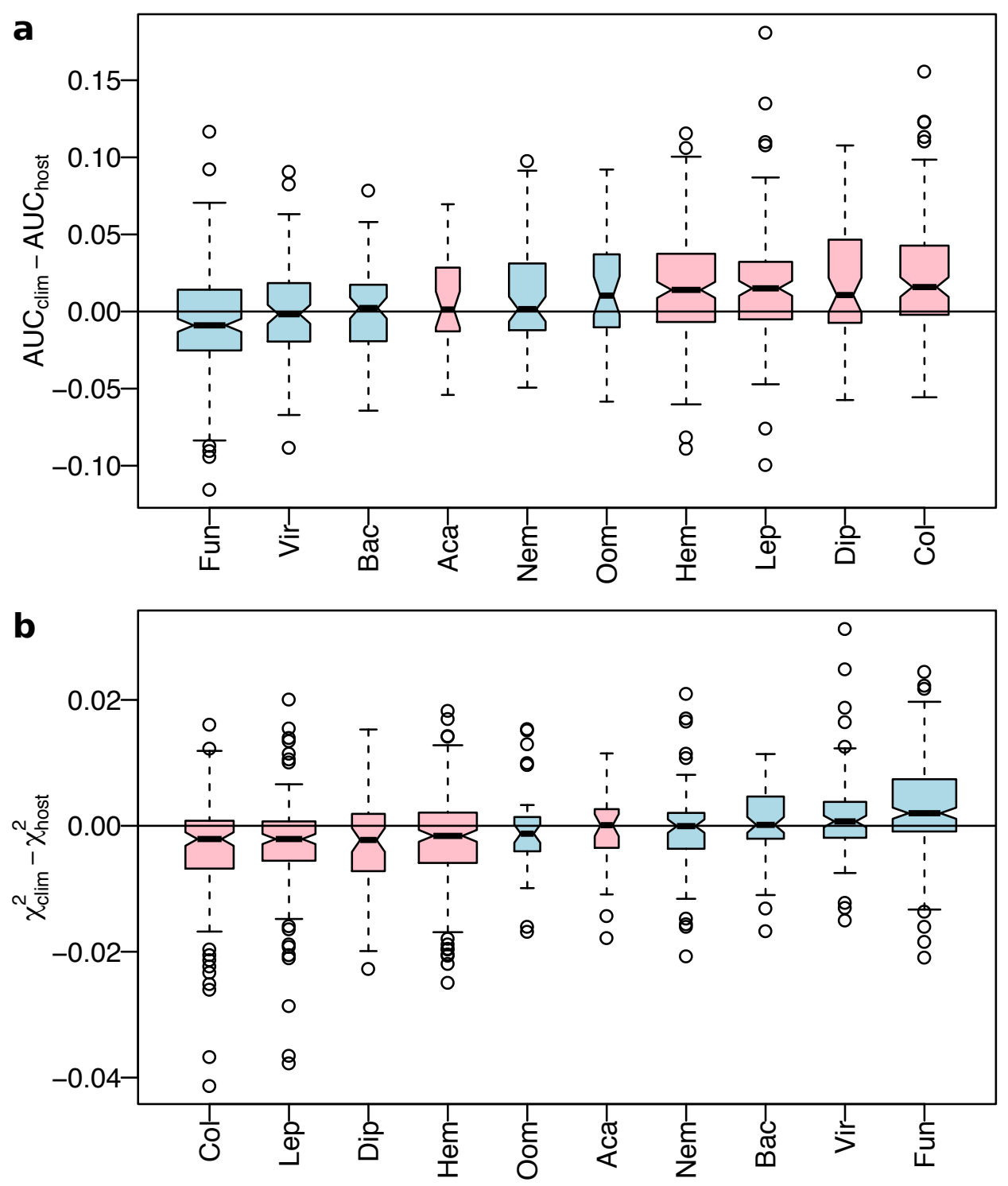

354

355 Supplementary Fig. 1. Explanatory power of climate vs. host models. a. AUC

356 statistic for models of CPP presence-absence adjusted for observational bias, for

357 models based on climatic variables $\left(\mathrm{AUC}_{\mathrm{clim}}\right) \mathrm{vs}$. host availability variables

358 (AUChost). Pathogen groups are blue, pest groups pink. The larger the value, the

359 better climatic variables predict CPP distribution. $\mathrm{AUC}_{\text {diff }}$ was greater for 
360 invertebrate pest distributions than pathogen distributions (mixed effects model

361 with random intercepts per taxonomic group, $\left.\mathrm{F}_{1,8}=12.3, \mathrm{p}=0.008\right)$. b. Chi-

362 squared statistic for models of CPP presence-absence adjusted for observational

363 bias, for models based on climatic variables $\left(\chi^{2}\right.$ clim $)$ vs. host availability variables

$364 \quad\left(\chi^{2}\right.$ host) $\cdot \chi^{2}$ diff was lower for invertebrate pest distributions than pathogen

365 distributions (mixed effects model with random intercepts per taxonomic group,

$\left.366 \mathrm{~F}_{1,8}=16.8, \mathrm{p}=0.004\right)$. Where notches in the boxes do not overlap between

367 categories, this indicates a significant difference among at the $5 \%$ level. Box

368 widths are proportional to the square root of sample size. 